\title{
Unification of psychophysical phenomena: The complete form of Fechner's law
}

\author{
KENNETH H. NORWICH and WILLY WONG \\ University of Toronto, Toronto, Ontario, Canada
}

\begin{abstract}
Many of the laws and empirical observations of fundamental psychophysics can be unified with a single equation, which has been called the complete form of Fechner's law. It can be shown that this law embraces both of the commonly used forms: Stevens's and Fechner's laws. It assumes one or the other form with appropriate values of the parameters. However, the complete equation confers an advantage beyond simply containing the classical laws. It offers greater flexibility in the representation of experimental data. It is shown that psychophysical phenomena may be represented by any number of triplets of quantities: subjective magnitude of stimulus, subjective just noticeable difference (jnd), and differential threshold. Each of the preceding quantities are functions of the physical magnitude of the stimulus. The investigator has the license to choose two of these quantities in the form he or she thinks is best; the third quantity is determined by the choice of the first two. Thus, for example, different forms of the law of sensation and different forms of the mathematical function for differential threshold may coexist with equal validity.
\end{abstract}

A primary aim of this paper is to show that when Fechner's law is written in its expanded or informational form, it bestows upon psychophysics a certain unity of character. That is, a number of ostensibly disparate phenomena can be related one to the other. This thesis will be demonstrated largely with respect to the sense of audition. Hence, the expanded form of Fechner's law will assume the form of a loudness function.

Fechner obtained the law that bears his name by assuming the validity of Weber's law, which stated that the fraction $\Delta I / I$, the intensity just noticeable difference ([jnd] or differential threshold) divided by the pedestal intensity, is equal to a constant. He further assumed that the subjective jnd, which we shall represent here by $\Delta L$, is also constant. Combining these two assumptions, Fechner was able to formulate a differential equation, which he then integrated or summated to give his famous law. By so doing, he had postulated that the law of sensation, which takes the general form of $L=f(I)$ (in our case, loudness is equal to some function of stimulus intensity), can be regarded as the sum of constituent jnds. Or, conversely, if

\footnotetext{
This research has been supported by an operating grant from the Natural Sciences and Engineering Research Council of Canada. W.W. has been supported by a University of Toronto Open Fellowship. K.H.N. is a member of the Institute of Biomedical Engineering, the Departments of Physiology and Physics at the University of Toronto. W.W. is a member of the Institute of Biomedical Engineering and the Department of Physics at the University of Toronto. The authors would like to thank Lawrence Marks, Lawrence Ward, Lester Krueger, and an anonymous reviewer for their help and guidance in the preparation of this paper. The authors are grateful to Alexander Aydt for his assistance in translating the work of A. Lehmann from the original German. Correspondence should be addressed to K. H. Norwich, University of Toronto, Institute of Biomedical Engineering, Toronto, ON, Canada M5S 3G9 (e-mail: k.norwich@utoronto.ca).
}

the loudness function is regarded in the Fechnerian manner as a sum of jnds, then, by differentiating this function, we obtain an expression for the Weber fraction.

In this paper, we shall reverse the order of activity. We shall begin with a form of the law of sensation (in this case, the loudness function), which we term the complete form of Fechner's law, and differentiate it to obtain the Weber fraction. However, the Weber fraction derived from the expanded form of Fechner's law is not exactly the one that Weber obtained. As intensity increases, the Weber fraction declines toward a plateau, which can be regarded as Weber's constant. It is, in fact, the type of curve measured by Riesz (1928), shown in Figure 1, for the differential threshold of the intensity of sound. Moreover, we shall not hold the subjective jnd to be constant, as Fechner did. Rather, we shal! permit it to vary as a function of stimulus intensity. None of these ideas are, in themselves, new. What is, perhaps, unfamiliar is the process of regarding variations in the law of sensation, the differential threshold and the subjective jnd together-that is, permitting these three quantities to change in unison.

The history of the " $1+\gamma I$ " variation on Fechner's law may extend back to the time of Fechner himself. Helmholtz (1856-1866/1924) suggested an expansion of Fechner's original law, as did Delboeuf (1873) (see Murray, 1993). The Delboeuf modification of Fechner's law assumed the form

$$
L=k \log \left(1+I / I_{n}\right)
$$

where $I_{n}$ is the result of internal neural activity.

We do not know the details of Delboeuf's derivation of Equation 1 (D. J. Murray, personal communication, August 1996; Nicolas, Murray, \& Farahmand, in press). However, one might derive his equation from Fechner's law in the following fashion: Beginning with 


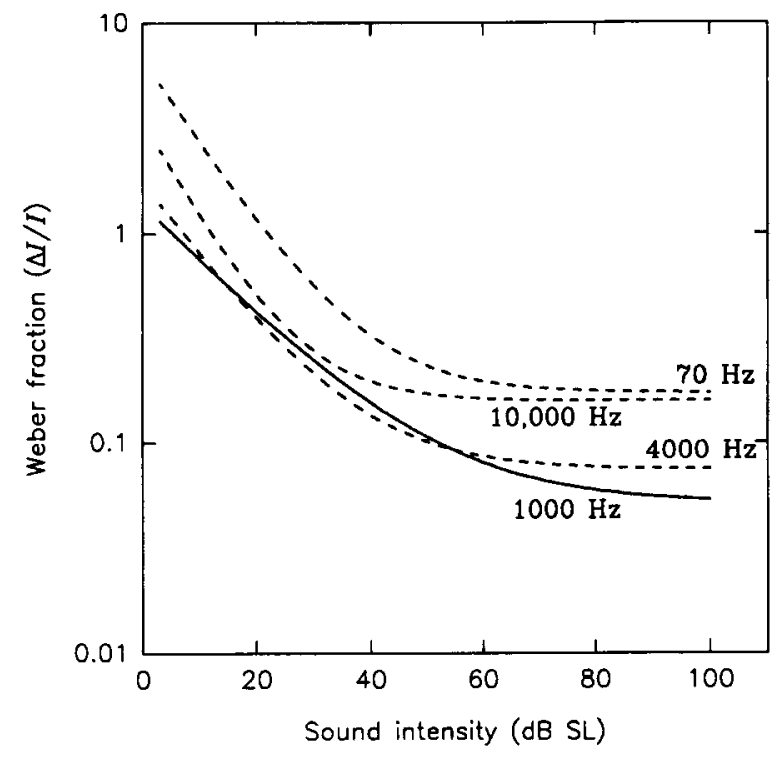

Figure 1. Weber fraction data of Riesz plotted at different frequencies using his empirical Equation 16 and parameters in Equations 17,18 , and 19. Intensity values are kept well below physiological saturation levels. Please see Appendix B with regard to sensation level.

$$
L=k \log \left(I_{\text {signal }}\right),
$$

we assume that $I_{\text {signal }}$ is actually a sum of two signals, the external signal, $I$, and the signal, $I_{n}$, due to "internal causes quite distinct from any external causes" (Nicolas et al., in press). Hence,

$$
L=k \log \left(I+I_{n}\right) .
$$

One problem with this equation is that the larger $I_{n}$ gets, the larger $L$ becomes, which is not correct. $I_{n}$ interferes with the subject's ability to sensate, so $L$ should not get larger. The other problem is that for $I=0, L \neq 0$. However, from experience, we know that loudness is zero in the absence of external signals. Hence, we might modify Equation 3 by subtracting off the loudness due to internal activity alone:

$$
\begin{aligned}
L & =k \log \left(I+I_{n}\right)-k \log \left(I_{n}\right) \\
& =k \log \left(\frac{I+I_{n}}{I_{n}}\right)=k \log \left(1+I / I_{n}\right) .
\end{aligned}
$$

Lehmann (1905) described Fechner's function

$$
\begin{aligned}
E & =c \log \left(R / R_{0}\right), \\
R_{0} & =\text { constant (e.g., absolute threshold) }
\end{aligned}
$$

as "incomplete" (in German unvollständig) and proposed the "complete" neurophysiological equation

$$
E=c \log \left(1+\frac{R}{x}\right)
$$

where $E$ is sensation (in German Empfindungen), $R$ is stimulus magnitude, $c$ and $x$ are constants $>0$. The same equation was later used by Békésy (1960) to account for the auditory Weber fraction data of Knudsen. Some elaboration on this historical material may be found in the Discussion section below.

The term "complete form of Fechner's law" was also the title of a paper by Nutting (1907). Nutting, in his analysis of the visual Weber fraction data of König, derived the following expanded form of Fechner's equation in large part empirically:

$$
L=L_{o} \ln \left[1+P_{m}\left(\left[\frac{I}{I_{\text {thresh }}}\right]^{n}-1\right)\right],
$$

where $L_{o}$ and $P_{m}$ are constants.

We shall see that the complete form of Fechner's law, when approximated for certain values of its parameters, gives rise to a power law. That is, if we regard an expression for summated jnds as a law of sensation, then the complete form of Fechner's law “contains" Stevens's law. Other parameter values will make the complete law look like Fechner's original logarithmic law. Thus, the complete form of the law can take on three countenances.

In Appendix A, we shall discuss the relationship between constant and nonconstant jnds and the loudness function. We shall also see that, by relaxing our criteria for a standard method of measuring sensation, many types of measured Weber fraction other than Riesz's can be brought under the umbrella of the complete Fechner law. For example, we can embrace the "near-miss" of McGill and Goldberg (1968).

Since loudness data conform more closely to the power law, we shall often use this form of the law. A simple calculation using the power function exponent as a function of frequency of tone will suffice to derive a theoretical set of equal loudness contours. These derived contours will be compared with the empirical contours measured by Robinson and Dadson (1956).

\section{THE COMPLETE FORM OF FECHNER'S LAW}

The complete form of Fechner's law is very similar to the one described by Nutting. We write it here in the form

$$
L=\frac{1}{2} k \ln \left(1+\gamma^{\prime} I^{n}\right),
$$

where $L$ is loudness, $I$ the intensity of sound expressed as power, and $k, \gamma^{\prime}$, and $n$ are constants. In recent years, we have been able to provide a physical interpretation of Equation 8 . The loudness, $L$, can be regarded as an entropythat is, as the information content of a tone of intensity, $I$. The factor of $1 / 2$ remains in the equation as a label from its informational heritage. The reader interested in the informational theory is referred to the recent monograph by Norwich (1993). However, for present purposes, we require only the mathematical form of Equation 8, not its informational interpretation.

One should not feel alienated by Equation 8 . It is, really, just an amalgam of the two familiar laws of sensa- 
tion, Fechner's and Stevens's. When $\gamma^{\prime} I^{n} \gg 1$, we see immediately that Equation 8 becomes

$$
L=\frac{1}{2} k n \ln I+\frac{1}{2} k \ln \gamma^{\prime},
$$

which is Fechner's law, since $1 / 2 k n$ and $1 / 2 k \ln \gamma^{\prime}$ are constants. When $\gamma^{\prime} I^{n} \ll 1$, a Taylor series expansion of Equation 8 leaves to first-order terms just

$$
L=\frac{1}{2} k \gamma^{\prime} I^{n},
$$

which is the usual power law of sensation championed by Stevens. The exponent, $n$, has often been assigned the value of 0.3 when $I$ is the intensity of a $1000-\mathrm{Hz}$ tone, expressed as power. Equation 8, the complete or parent law of sensation, embraces collectively the two daughter laws.

\section{INTRODUCING THE THRESHOLD OF HEARING}

If we choose to express sound intensity in units of decibel sensation level (SL), it might be convenient to rewrite Equation 8 as

$$
L=\frac{1}{2} k \ln \left[1+\gamma\left(\frac{I}{I_{\text {thresh }}}\right)^{n}\right],
$$

where $I_{\text {thresh }}$ is the threshold intensity, and $\gamma$ is a new constant.

By assumption, $k$ is a constant, independent of both intensity and frequency. However, the parameters $\gamma, n$, and $I_{\text {thresh }}$ are all frequency dependent, as we shall see in the following section.

The loudness equation is valid only for sound intensity above threshold and below physiological saturation levels. To account for effects near threshold, we introduce a small correction to Equation 11 . Since no response is possible until a threshold is reached, we might incorporate the threshold in the following manner:

$$
L= \begin{cases}L-L_{\text {thresh }}, & L>L_{\text {thresh }}, \\ 0, & \text { otherwise }\end{cases}
$$

where $L_{\text {thresh }}$, the loudness threshold, is obtained from Equation 11 by setting $I=I_{\text {thresh }}$. Combining Equations 11 and 12 , we can now write for the loudness function

$$
\begin{aligned}
L & =\frac{1}{2} k \ln \left[1+\gamma\left(\frac{I}{I_{\text {thresh }}}\right)^{n}\right]-\frac{1}{2} k \ln (1+\gamma) \\
& =\frac{1}{2} k \ln \left[\frac{1+\gamma\left(\frac{I}{I_{\text {thresh }}}\right)^{n}}{1+y}\right],
\end{aligned}
$$

for $\geq I_{\text {thresh. }}$.

When $I \rightarrow I_{\text {thresh }}, L \rightarrow 0$, as required. Notice that no new parameters are added to the equation - the loudness threshold is obtained in terms of the existing parameters $k$ and $\gamma$. Threshold has been treated deterministically here in the interests of obtaining a simple function for $L$. More realistically, threshold must be represented probabilistically, perhaps using signal detection theory, but leading to a considerable increase in complexity.

In Figure 2, we have plotted the loudness function given by Equation 13, with parameter values $k=997.4, \gamma=$ $1.861 \times 10^{-4}$, and $n=0.27$, together with the measured loudness data of R. P. Hellman and Zwislocki (1961). It may be seen that the theoretical loudness function conforms very closely to the measured data.

\section{INTENSITY DISCRIMINATION}

Differentiating the loudness function, Equation 13, we obtain

$$
\frac{d L}{d I}=\frac{1}{2} k \frac{\gamma n I^{n-1} I_{\text {thresh }}^{-n}}{1+\gamma\left(\frac{I}{I_{\text {thresh }}}\right)^{n}} .
$$

Rearranging for $d I / I$, and replacing the differential quantities by their respective finite differences, we obtain

$$
\frac{\Delta I}{I}=\frac{2 \Delta L}{n k}\left[1+\frac{1}{\gamma}\left(\frac{I_{\text {thresh }}}{I}\right)^{n}\right] .
$$

This step is a good approximation if $\Delta I / I \ll 1$. Experimental data show that this approximation holds for large $I$ but weakens as $I$ approaches the threshold.

It is important to note that Equation 15 can be derived from either Equation 11 or Equation 13, since the two equations differ only by an additive constant-namely, $-1 / 2 k \ln (1+\gamma)$.

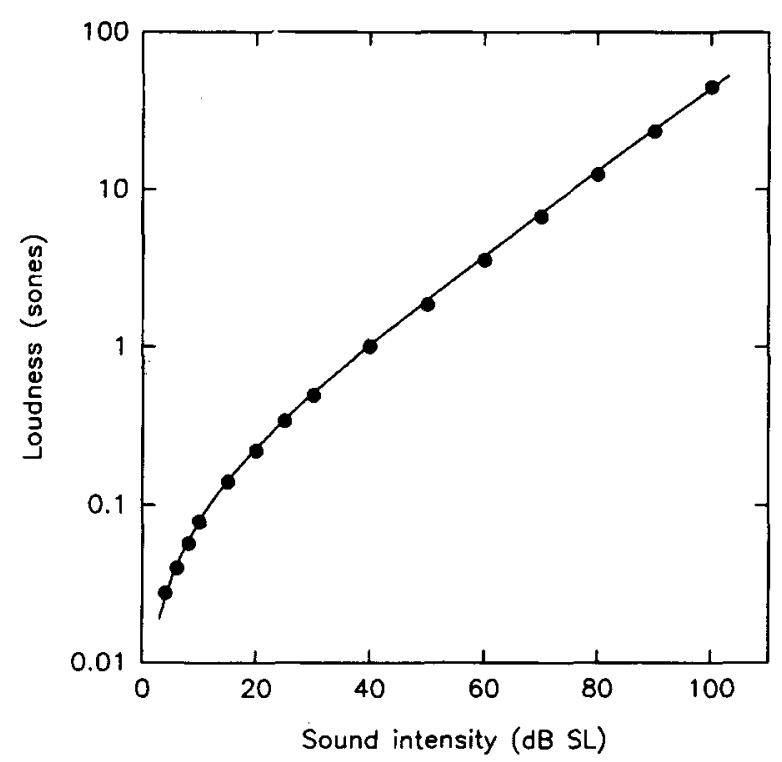

Figure 2. The loudness data of Hellman and Zwislocki (1961) as fitted by the loudness Equation 13 (smooth curve). See Appendix $B$ with respect to sensation level. 
On the left side of Equation 15 is the fractional change in sound intensity; hence, Equation 15 is an expression of the Weber fraction. Since $\Delta L$ represents a change in loudness sensation resulting from the intensity increment, we can therefore take $\Delta I$ as measuring the change in sound intensity required to produce one jnd in loudness $\Delta L$.

Utilizing Fechner's conjecture of the constancy of the loudness jnd (constant with respect to variations in sound intensity), we set $\Delta L$ constant (please see Appendix A). Rewriting Equation 15 by setting $S_{\infty}=2 \Delta L / n k$, and $\left(S_{o}-\right.$ $\left.S_{\infty}\right)=2 \Delta L / n k \gamma$, both of which are constant for a given frequency, we obtain

$$
\frac{\Delta I}{I}=S_{\infty}+\left(S_{o}-S_{\infty}\right)\left(\frac{I_{\text {thresh }}}{I}\right)^{n},
$$

where $S_{o}-S_{\infty}$ must exceed zero, since $2 \Delta L / n k \gamma$ consists only of factors greater than zero.

This equation is now identical in form to the empirical equation used by Riesz (1928) to fit his Weber fraction data and is similar to the expression derived by Siebert (1968) using a model based on single auditory nerve fiber activity. The Weber fraction takes on the value $S_{o}$ when $I$ assumes its threshold value and approaches $S_{\infty}$ as $I$ becomes large. As Equation 16 shows, $\Delta I / I$ starts high (i.e., $S_{o}$ ) for small $I$ values and progressively decreases toward a lower asymptote or plateau (i.e., $S_{\infty}$ ) as $I$ increases.

Riesz used the method of beats or amplitude modulation to measure the Weber fraction. Although his results have differed from measurements made by other experimental techniques, Riesz's experiments have been validated by Harris (1963) and more recently by Ward and Davidson (1993).

Riesz measured Weber fraction curves for different frequencies, and he expressed his three constants $S_{o}, S_{\infty}$, and $n$ as empirical functions of frequency, $f$,

$$
\begin{aligned}
S_{\infty} & =0.000015 f+\frac{126}{80 f^{0.5}+f}, \\
S_{o} & =0.3+0.0003 f+\frac{193}{f^{0.8}}, \\
n & =\frac{244,000}{\left(358,000 f^{0.125}+f^{2}\right)}+\frac{0.65 f}{(3,500+f)} .
\end{aligned}
$$

Riesz used the symbol $r$ in place of our $n$. These three empirical parameters encode the measured dependency of the Weber fraction on frequency. Therefore, when introduced into the loudness function, which is the integrated form of the Weber fraction, these same three parameters encode the dependency of loudness on frequency- a dependence that may be used to obtain equal loudness contours.

The exponent $n$ in Equation 16, which has previously been identified with the power function exponent of loudness, can now be checked for numerical consistency. For the $1000-\mathrm{Hz}$ curve, Riesz obtained a value of 0.2764 , which compares favorably with the exponents obtained from $1000-\mathrm{Hz}$ loudness data. For larger values of frequency,
Riesz's values differ somewhat from values of $n$ measured by other methods.

Rewriting Equation 15 as

$$
\frac{\Delta I}{I}=\frac{2 \Delta L}{n k}+\frac{2 \Delta L}{n k \gamma}\left(\frac{I_{\text {thresh }}}{I}\right)^{n},
$$

and comparing Equation 20 term by term with Equation 16, we see that

$$
\Delta L=\frac{n k S_{\infty}}{2}
$$

and

$$
\gamma=\frac{S_{\infty}}{S_{o}-S_{\infty}} .
$$

$\Delta L$ is the magnitude of the loudness jnd. Since, by assumption, $k$ is a constant independent of frequency, while $n$ and $S_{\infty}$ depend on frequency, it may therefore be seen from Equation 21 that, when interpreted by Riesz's measurements, $\Delta L$ changes with frequency. In Figure 3, we plot $\Delta L / k$ and $\gamma$ as functions of frequency. Notice that the loudness jnd is smallest in the $1-2 \mathrm{kHz}$ region, as might have been expected, since human hearing is most sensitive in that region.

\section{TOWARD THE UNIFICATION OF PSYCHOPHYSICS}

Psychophysics currently enjoys a multiplicity of functional forms representing what are ostensibly the same psychophysical function. The examples below are drawn from the literature on audition.

\section{Weber Fraction}

Riesz (1928), as we have seen, measured Weber fractions that decline with increasing stimulus intensity to a plateau or constant value. McGill and Goldberg (1968) in their well-known "near-miss" paper found that the Weber fraction declined as intensity to the power 0.095 but did not plateau. That is, $\Delta I / I^{0.905}=$ constant. Jesteadt, Wier, and Green (1977) reported various measures of the Weber fractions that declined monotonically with powers as high as about 0.13 . That is, $\Delta I / I^{0.87}=$ constant. Different experimental techniques will generate different forms of the Weber fraction curve (e.g., Viemeister, 1988). Different statistical criteria for measuring the jnd will produce different magnitudes of the differential threshold, $\Delta I$, and, hence, different values for the Weber fraction.

\section{Loudness}

The standard loudness curve endorsed by ISO R1311959 (e.g., Scharf, 1978) is similar to the one shown in Figure 2. It is usually prepared by the method of magnitude estimation and production and is related to the sone scale of loudness. However, there is a good deal of intersubject variability, as seen particularly in the data of McGill (1960). Recently, West (1996), West and Ward 

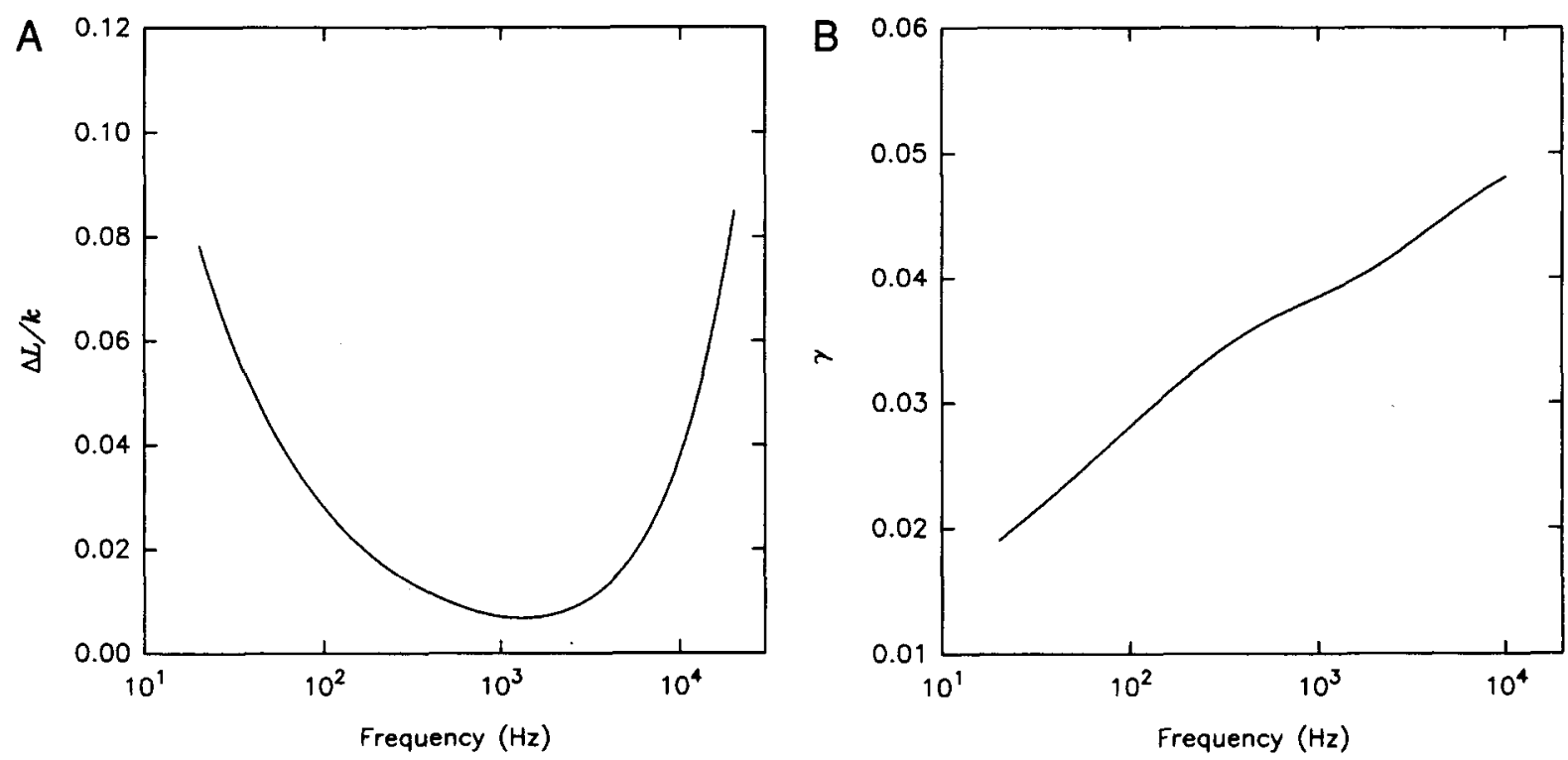

Figure 3. (A) Plotting the loudness jnd as a function of frequency from the data of Riesz (1928). Riesz's data have been interpreted as derivatives of the loudness function, providing the value of the jnd from Equation 21 . By assumption, $k$ is a constant independent of both intensity and frequency. The function shows a minimum at 1-2 kHz, corresponding to the most sensitive region of normal human hearing. (B) Using the data of Riesz, $\gamma$ from Equation 22 is plotted. Notice that $\gamma \ll 1$ throughout the entire frequency range. $\hat{\gamma}$ (i.e., $\gamma$ at $1000 \mathrm{~Hz}$ ) never differs from $\gamma_{\max }$ or $\gamma_{\min }$ by a factor greater than 2.5 .

(1994), and Marks, Galanter, and Baird (1995), following up on earlier investigators, have demonstrated that subjects can be trained to produce loudness curves that conform to a specified format. That is, for tones of $1000 \mathrm{~Hz}$, subjects may produce an excellent loudness curve with an exponent half or double the standard value of about 0.3 . These constrained curves are, however, perfectly legitimate maps of loudness onto sound intensity.

\section{Magnitude of the Subjective jnd}

Fechner assumed that the subjective jnd, represented here by $\Delta L$, was constant for all loudness levels. However, Fechner's conjecture has been tested quantitatively by many investigators in recent years beginning, perhaps, with Stevens in 1936, who calculated that the magnitude of the jnd increased with increasing loudness (as the 1.2 power of the cumulative number of jnds above threshold [please see also Appendix A and Discussion section]). More recently, Krueger (1989) again raised this possibility, and W. S. Hellman and R. P. Hellman (1990) and others have calculated that the subjective jnd increases as the square root of loudness. ${ }^{1}$

A degree of unity can be conferred on the world of psychophysics, however, if we recognize that the Weber fraction (or differential threshold, $\Delta I$ ), loudness, $L$, and subjective jnd, $\Delta L$, are interrelated. If each of these quantities is regarded as a function of only the variable, $I$, then the three quantities form sets of three related quantities, any two of which imply the third. To take a simple example from physics, one may define the unit of length and the unit of time by taking any two of the following three standards: a standard unit of length, a standard unit of time, and a standard speed of light in vacuo. For example, given the standard meter and the standard second, we can specify the speed of light in meters per second; or, given the speed of light in meters per second and the standard second, we can specify the length of the standard meter.

A key to the formulation of consistent sets of the three quantities, $L, \Delta L$, and $\Delta I$, may be the complete form of Fechner's law, Equation 13 and its differential forms, Equation 14 and Equation 15. There are three possible combinations of the three quantities. Given $L$ (i.e., the parameters $k, n, \gamma$, and $I_{\text {thresh }}$ in Equation 13, and $\Delta L$, we can find $\Delta I$ using Equation 15 . Given $L$ and $\Delta I$, we can find $\Delta L$ using Equation 15. Given $\Delta I$ and $\Delta L$ as functions of $I$, we can find $L$ by substituting these values into Equation 15 and curve-fitting for the parameters $k, n$, and so on. That is, any two quantities imply the third. However, to use this unifying procedure universally, we must relax our demands for a fixed form of the loudness function. The loudness function will have to be permitted to vary within certain bounds. These bounds seem to be constrained within the expanded or complete Fechner law. That is, we know of no loudness data reported that are not encompassed by the expanded law, and we are aware of no simpler law that will contain all reported data.

\section{Six Cases}

We have constructed Table 1 to give a few examples of an infinite set of triplets consistent with the complete form of Fechner's law. This table is analogous to Figure 4.3 of Baird and Noma (1978), which they devised to elaborate the "fourfold way" in psychophysics. Their fourfold way involves $\Delta L$ and $\Delta I$ (columns 3 and 4 of Table 1 ), treating each as a constant or as a variable (dependent on either 
Table 1

Summary of the "Critical Triplets" Discussed in the Paper

\begin{tabular}{|c|c|c|c|}
\hline & $\begin{array}{c}L \\
\text { (Loudness function) }\end{array}$ & $\begin{array}{c}\Delta L \\
\text { (Loudness jnd) }\end{array}$ & $\frac{\frac{\Delta I}{I}}{\text { (Weber fraction) }}$ \\
\hline 1 (Fechner) & $\begin{array}{c}L=A \log I+B \\
\text { (Incomplete form of Fechner's law) }\end{array}$ & $\begin{array}{c}\Delta L=C=\text { constant } \\
\text { (Fechner's assumption) }\end{array}$ & $\begin{array}{c}\frac{\Delta I}{I}=\frac{C}{A}=\text { constant } \\
\text { (Weber's law) }\end{array}$ \\
\hline 2 & $\begin{array}{l}\quad L=\frac{1}{2} \ln \left(\frac{1+\gamma\left(I / I_{\text {thresh }}\right)^{n}}{1+\gamma}\right) \\
\text { (Complete form of Fechner's law) }\end{array}$ & $\begin{array}{c}\Delta L=\text { constant } \\
\text { (Fechner's assumption) }\end{array}$ & $\begin{aligned} \frac{\Delta I}{I}= & \frac{2 \Delta L}{n k}\left[1+\frac{1}{\gamma}\left(\frac{I_{\text {thresh }}}{I}\right)^{n}\right] \\
& (\text { Riesz, 1928) }\end{aligned}$ \\
\hline 3 & $\begin{array}{c}L=\frac{1}{2} \gamma^{\prime} I^{n} \\
\text { (Power law) }\end{array}$ & $\begin{array}{c}\Delta L \propto L^{1 / 2} \\
(\text { Hellman \& Hellman, 1990) }\end{array}$ & $\begin{array}{c}\frac{\Delta I}{I} \propto \frac{1}{I^{n / 2}} \\
\text { (Jestead et al., 1977) }\end{array}$ \\
\hline 4 & $\begin{array}{l}L=\frac{1}{2} \gamma^{\prime} I^{3} \\
\text { (Power law) }\end{array}$ & $\Delta L \propto L^{2 / 3}$ & $\begin{array}{c}\frac{\Delta I}{I} \propto \frac{1}{I^{0.095}} \\
(\text { McGill \& Goldberg, 1968) }\end{array}$ \\
\hline 5 & $\begin{array}{c}L=\frac{1}{2} \gamma^{\prime} I^{n}(n \text { arbitrary) } \\
\text { (Power law) }\end{array}$ & $\begin{array}{c}\Delta L \propto L \\
\text { (Ekman's principle, 1959) }\end{array}$ & $\begin{array}{l}\frac{\Delta I}{I}=\text { constant } \\
\text { (Weber's law) }\end{array}$ \\
\hline 6 & $\begin{array}{c}L \propto I^{(1-b)} \\
(\text { Power law) }\end{array}$ & $\begin{array}{c}\Delta L=\text { constant } \\
\text { (Fechner's assumption) }\end{array}$ & $\begin{array}{c}\frac{\Delta I}{I} \propto \frac{1}{I^{n / 2}} \\
\text { (Near-miss to Fechner's law) }\end{array}$ \\
\hline
\end{tabular}

Note - Each row corresponds to a consistent set of relationships - that is, given any two elements from one row, the third element is implied.

$L$ or $I$ ) and generating a consistent third member - that is, the law of sensation (column 2).

Row 1. We may observe that Fechner was the first to have carried out this procedure of "two implies the third." Fechner assumed Weber's law, $\Delta I / I=$ constant, and assumed the constancy of $\Delta L$. He then derived a consistent third member, $L$, by integration. The third member is, of course, the classical Fechner law (Equation 9), which we may now regard as a special or restricted form of the complete Fechner law.

Row 2. We have examined a second consistent set of $L, \Delta L$, and $\Delta I$ : the set derived from Riesz's work. $L$ was given by the complete form of Fechner's law, Equation 13, $\Delta I$ issued from Equation 15, which Riesz measured, and $\Delta L$ was taken as constant, to complete the consistent set.

Row 3. Let us examine a third consistent set. Taking $L$ from Stevens's law, now obtained as a special case of the complete form of Fechner's law, Equation 10, and choosing $\Delta L$ freely (see note 1 ), we set $\Delta L \propto L^{1 / 2}$. That is, we replace Fechner's conjecture by setting the subjective jnd proportional to the square root of loudness, as proposed by several investigators. The third member of the set, $\Delta I$, is determined by our selection of the first two members. We obtain the Weber fraction by differentiating $L$ as before:

$$
\frac{\Delta I}{I}=\frac{2 \Delta L}{\gamma n k}\left(\frac{I_{\text {thresh }}}{I}\right)^{n} \text {. }
$$

Inserting the condition that $\Delta L \propto L^{1 / 2}$, and introducing $L$ from Equation 10, we find

$$
\frac{\Delta I}{I} \propto \frac{1}{I^{n / 2}} .
$$

Thus, we see ${ }^{2}$ that when the exponent, $n$, in Stevens's law, Equation 10, takes on the value 0.27 , the Weber fraction will decline with increasing $I$ raised to the exponent $n / 2$, or about 0.135 . This behavior is consistent with the differential thresholds of Schacknow and Raab (1973) $(n=$ 0.125 ) as reported by Jesteadt et al. (1977). Given two members of the set of $L, \Delta L$ and $\Delta I$, we have found the third member, consistent with the complete form of Fechner's law.

Row 4. Now, let us find a consistent set for the near-miss law of McGill and Goldberg (1968). These investigators found that

$$
\Delta I \propto I^{0.905} .
$$

An exponent of 1 (rather than 0.905 ) would have given Weber's law-hence, the near-miss. Since we are at liberty to choose a loudness function, we may choose Stevens's law as the particular case of the complete Fechner law. That is,

$$
L \propto I^{0.3}, \text { or } I \propto L^{\frac{1}{0.3}} .
$$

Differentiating and so on,

$$
\frac{\Delta L}{\Delta I} \propto I^{(0.3-1.0)}=I^{-0.7} .
$$

Introducing Equation 25,

$$
\begin{aligned}
\Delta L & \propto I^{-0.7} \Delta I \\
& \propto I^{-0.7} I^{0.905}=I^{0.205},
\end{aligned}
$$

and, finally, using Equation 26,

$$
\Delta L \propto\left[L^{\frac{1}{0.3}}\right]^{0.205}=L^{0.683} .
$$

That is, the subjective jnd varies approximately as the $2 / 3$ power of loudness (see note 1). $\Delta I$ from Equation 25, $\Delta L$ from Equation 29, and $L$ from Equation 26 constitute a consistent set. 
Row 5. Suppose now that we take $\Delta L$ proportional to $L$ (the Brentano-Ekman-Teghtsoonian principle; Ekman, 1959; Krueger, 1989). We shall preserve Weber's law by keeping $\Delta I / I$ constant. Beginning with Weber's law,

$$
\Delta I \propto I,
$$

we append

$$
\Delta L \propto L
$$

Dividing the two equations gives

$$
\frac{\Delta L}{L} \propto \frac{\Delta I}{I},
$$

which, when integrated, gives $\ln L \propto n \ln I+$ constant, from which Equation 10, the simple power law of sensation, follows directly. That is, Weber's law is preserved with the simple power law of sensation.

Row 6. Finally, we consider the case advocated by Krueger (1989). We let $L$ assume the form

$$
L \propto I^{1-b}, b=\text { constant }
$$

and

$$
\frac{\Delta I}{I} \propto I^{-(1-b)} .
$$

The exponents of $I$ in the latter two equations are equal in magnitude and opposite in sign, a combination that is designed to preserve Fechner's conjecture. That is, differentiating Equation 33,

$$
\Delta L \propto I^{-b} \Delta I .
$$

From Equation 34, $\Delta I \propto I^{b}$. Substituting $\Delta I \propto I^{b}$ into Equation 35 gives

$$
\Delta L=\text { constant }
$$

That is, Fechner's conjecture is preserved together with the power law of sensation.

It may be seen that Fechner's conjecture (i.e., that $\Delta L$ [column 3] is a constant) will always be preserved if the derivative of $L(d L / d I$ [column 2]) times the value of $\Delta I$ (column 4$)$ is a constant. Thus, in row $1, A / I(=$ derivative of $L) \times$ constant $\times I(=\Delta I)$ is a constant; in row $6, I^{-b}$ (= derivative of $L) \times$ constant $\times I^{b}(=\Delta I)$ likewise is a constant. It should be noted too that, to fulfill Fechner's conjecture, the exponent for the power function in column 2 must match the exponent shown in column 4 (e.g., $1-b$ in row 6 ); it is no longer free to take other values, as was the case with rows 4 and 5 .

\section{EQUAL LOUDNESS CONTOURS}

Riesz proceeded to integrate or summate the jnds he had measured and obtained a theoretical set of equal loudness contours by matching ratios of sums of jnds. We shall proceed somewhat differently by matching directly the loudness of two pure tones of differing frequencies. The experimental procedure is straightforward. Measurements may be made in free field or using head- phones. We shall regard them as made in a free field for later comparison with the equal loudness contours measured by Robinson and Dadson. A comparison tone of frequency $f$ is adjusted in intensity $I$, until it sounds as loud as the reference tone of intensity $\hat{I}$ and frequency $\hat{f}$ (usually set at $1000 \mathrm{~Hz}$ ). The frequency of the reference tone is fixed, whereas $I$ is varied as a parameter (usually set at $10,20,30 \ldots d B$ ).

Using Equation 13, we can now match the loudness of two tones, differing in both frequency and intensity, with a loudness balance condition of the following form:

$$
L(I, f)=L(I, 1000 \mathrm{~Hz}) .
$$

Equation 37 can be solved explicitly for $I$ as a function of $f$ with $I$ as a parameter. Solved exactly, the resulting equation is an unwieldy expression but is conceptually no different from Equation 37: loudness at one frequency equals loudness at another.

A good approximation of Equation 37 may be obtained by observing that $\gamma \ll 1$, since the complete form of Fechner's law reduces to a simple power function for audition, as shown in Equation 10. Substituting Equation 13 into Equation 37 and using the inequality $\gamma \ll 1$, we obtain the simple form

$$
\gamma\left(\frac{I}{I_{\text {thresh }}}\right)^{n}=\hat{\gamma}\left(\frac{I}{\hat{I}_{\text {thresh }}}\right)^{\hat{n}} .
$$

The values of $\gamma, n, I_{\text {thresh }}$, and $I$ on the left side of this equation refer to the comparison tone of any desired frequency, $f$. The values of $\hat{\gamma}, \hat{n}, \hat{I}_{\text {thresh }}$, and I on the right side refer to the reference tone at $1000 \mathrm{~Hz}$.

We can further simplify this equation with the help of Figure $3 \mathrm{~B}$. Notice that the ratio $\hat{\gamma} / \gamma$ never changes by a factor of more than 2.5. Since we are evaluating the logarithm of Equation 38 to calculate the contours, we see that the $\log$ of $\hat{\gamma} / \gamma$ is approximately zero (since the ratio is close to one), and we can now replace Equation 38 with

$$
\log _{10} \frac{I}{I_{\text {thresh }}} \simeq \log _{10}\left(\frac{I}{\hat{I}_{\text {thresh }}}\right)^{\hat{n} / n} .
$$

That is, the contours depend largely on the single parameter $n$, in the ratio $\hat{n} / n$. Recalling that $n(f)$ is the exponent in the usual power function for loudness, we choose a function similar to the one suggested by Marks (1974b, Equation 3.4, p. 74),

$$
n(f)=0.28+2.17 f^{-0.59}+0.01 f^{0.2},
$$

which is plotted in Figure 4.

When the contours are derived from Equation 37, with $n$ taken from Equation 40, we can calculate a set of equal loudness contours in decibel SL (i.e., relative to threshold) against frequency. The theoretical contours are shown in Figure 5A. We must add to these curves the threshold data of Robinson and Dadson (1956) (please see Appen$\operatorname{dix} B$ ). The result is a theoretical set of equal loudness contours in decibel SPL (i.e., relative to $10^{-16} \mathrm{~W} / \mathrm{m}^{2}$ ), 


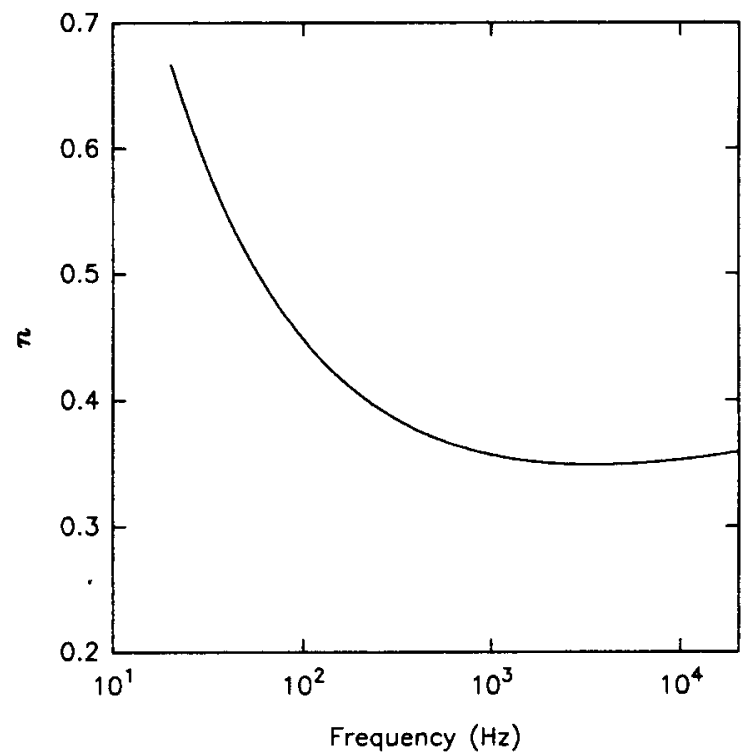

Figure 4. From Equation 40, the exponent is plotted as a function of frequency.

shown in Figure 5B. The derived equal loudness contours match the measured contours quite precisely.

\section{DISCUSSION}

The particular form of the expanded Fechner law, Equations 8 and 13, emerged from an entropic or informational theory of sensation and perception (Norwich, 1993; Norwich \& Wong, 1995). It is instructive to note that the completion or expansion of Fechner's law in this case was effected by compounding a noise or "reference signal" with the pure stimulus signal and is, in this respect, parallel to the approach of Delboeuf. In place of the Delboeuf's $1+I / I_{n}$, we have $1+\gamma^{\prime} I^{n}$. The factor $I_{n}$ in Delboeuf's equation is associated with the quantity $\gamma^{\prime}-1 / I_{\text {ref }}$ in Equation 8 , where $I_{\text {ref }}$ is the reference signal generated by the sensory organ (Norwich, 1993). An appeal of the complete form of Fechner's law is that it produces an improved fit to experimental data, and it avoids what has been termed negative sensations (Murray, 1993). That is, if $R<R_{0}$ in Equation 5, the incomplete form of the law, $E$ becomes negative. $E$ cannot be negative in the complete law.

We showed that the complete form of Fechner's law contained or embraced the two standard forms of the law of sensation: the classical form of Fechner's law, and the power law. Expressed in another way, the complete law, subject to certain values of its parameters, could be written more simply as either the semilog law or the power law of sensation, with little loss in accuracy. Certain modalities of sensation, such as audition, favor the power law more strongly.

The expanded form of Fechner's law with stimulus intensity represented to the first power $(n=1)$ seems to have been suggested by Helmholtz (1856-1866/1924) for the sense of vision. He reasoned as follows (representing brightness by $E$ and changing Helmholtz's symbol for stimulus intensity to $I$ to match our own): Fechner's differential equation assumed the well-known form

$$
d E=A \frac{d I}{I},
$$

with $A$ constant, whose solution is the classical or incomplete Fechner law of sensation. But, reasoned Helmholtz,

the influence of the intrinsic light of the eye must make itself felt. Together with the stimulation due to external light,
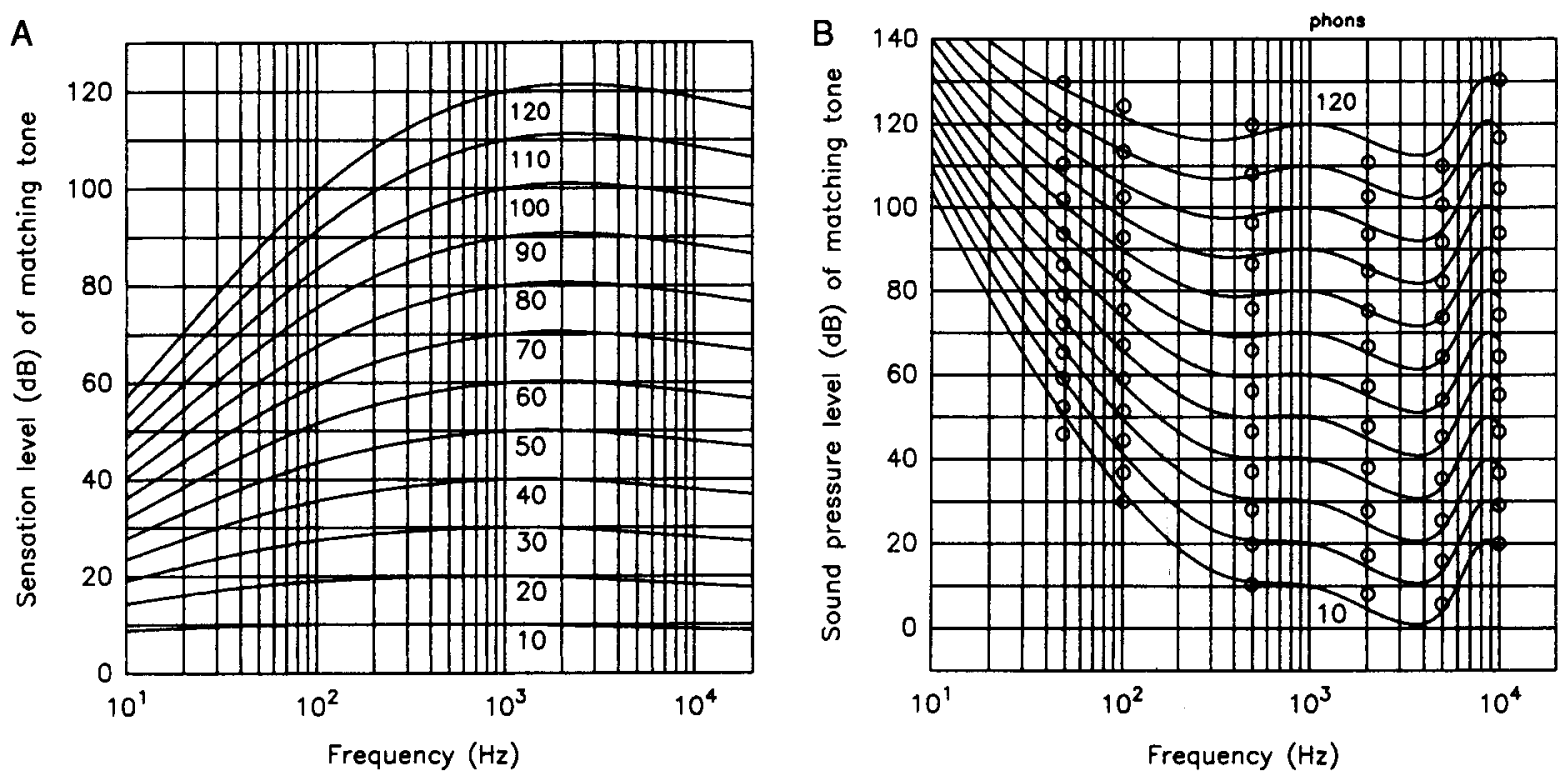

Figure 5. (A) Equal loudness contours derived using the loudness balance function, Equation 37, with $n(f)$ calculated from Equation 40. Decibels (dB) SL are plotted against tone frequency. (B) The contours as shown in panel A are replotted in dB SPL. The threshold data were supplied by the lowest contour of Robinson and Dadson (1956). The derived curves are compared with the measured values of Robinson and Dadson (open circles). 
there is always besides a stimulation due to internal causes, the amount of which may be considered as being equivalent to the stimulation by a light of luminosity $I_{n}$. It would be more accurate, therefore, to write the formula for the least perceptible degrees of the intensity of sensation as follows:

$$
d E=A \frac{d I}{I+I_{n}} .
$$

This differential equation, then, replaces the above equation by Fechner. Solving Helmholtz's Equation 42, we obtain

$$
E=A \log \left(I_{n}+I\right)+C .
$$

For completeness, then, we include the entropic or informational differential equation, which gives rise to the complete form of Fechner's law with $I$ raised to the power $n$ (Norwich, 1993):

$$
d E=\frac{1}{2} k n\left(1-e^{-2 E / k}\right) \frac{d I}{I} .
$$

When this differential equation is integrated with $L$ set equal to $E$, we obtain the complete law in the form of Equation 8. We may observe that the constant $\gamma^{\prime}$ does not appear in the differential equation but enters the solution to the differential equation as a constant of integration. Recall that this equation, as well as Helmholtz's, is derived from considerations of an internally generated stimulus signal. Note that, when $E$ is large, the entropy Equation 44 approaches Fechner's original Equation 41.

Lehmann's description of Fechner's law as incomplete was based on his own chemical theory of nerve excitation. Translating from the German in Lehmann's introduction:

Fechner's measurement formula [Massformel] $E=c \log$ $\left(R / R_{0}\right)$ is incomplete, being valid only for those cases where action of the nervous organs consume only a small amount of material, so that one does not have to go to [the substance's] limit ... Where nervous activity, on the contrary, uses more substance, for example with photochemical processes in the retina, it is necessary to allow metabolism to proceed to completion, and in so doing one obtains a more complete psychophysical formula ...

There follows an equation slightly more complicated than the equation above. The simpler form is derived in Lehmann (1905, p. 21). We include this brief description of Lehmann's work in the interests of completeness and historical precedent.

Invoking the findings of King and Lockhead (1981), Koh and Meyer (1991), West and Ward (1994), Marks et al. (1995), and West (1996), who showed that subjects could learn to produce different subjective responses (e.g., generate loudness curves that are power functions with various prescribed values of the exponent, $n$ ), we suggested that a spectrum of loudness curves might be considered as legitimate representations of the sensation of loudness. We suggested that, at a given frequency, three quantitiesloudness, $L$, the differential threshold for sound intensity, $\Delta I$, and the differential threshold for loudness, $\Delta L$-can vary only in unison. All three of these quantities are functions of intensity, $I$. The investigator is at liberty to choose or measure any two of these quantities in accordance with his or her view of psychophysics, but the third quantity is then determined by the selection of the first two.

The reader might think that this restriction (two determine the third) is artificial and can be ignored. For example, suppose that $L$ and $\Delta L$ are chosen by the investigator in accordance with his/her view of nature. He/she might then think that the differential threshold can be measured by an independent experiment. However, $\Delta I$ is now predetermined. Using the definition of derivative, we have, approximately,

$$
\Delta I=\Delta L / \frac{d L}{d I} .
$$

Thus, $\Delta I$ (as a function of $I$ ) is now determined as the ratio of $\Delta L$ (a constant or a function of $I$ ) to the derivative of $L$ with respect to $I$. That is, the Weber fraction cannot now be measured independently. Any two of $L, \Delta L$, and $\Delta I$ as functions of $I$ determine the third.

We have been concerned primarily with the issue of consistency. If Investigators $A$ and $B$ obtain two different measures of the differential threshold, $(\Delta I)_{\mathrm{A}}$ and $(\Delta I)_{\mathrm{B}}$, we have accepted the validity of both measures. Each measure is presumed to be true to the methodology and statistical assumptions employed by the investigator. We have been concerned only with ascertaining the matching values of the remaining variables, such that $\left\{(\Delta I)_{\mathrm{A}},(\Delta L)_{\mathrm{A}}\right.$, $\left.L_{\mathrm{A}}\right\}$ and $\left\{(\Delta I)_{\mathrm{B}},(\Delta L)_{\mathrm{B}}, L_{\mathrm{B}}\right\}$ constitute two consistent sets. The complete Fechner law provides us with the requisite flexibility to achieve these consistent sets.

It is important to appreciate the connection between the complete form of Fechner's law and the triplet-ofvariables concept. Because the observed or empirical loudness-intensity relation is pleomorphic, so to speak (taking on many forms), we can understand why different mathematical functions for $\Delta L$ and $\Delta I$ can remain consistent with different but equally legitimate forms of the loudness-intensity function.

One might ask what the results would be if an inconsistent triplet were used - that is, one of the three quantities $L, \Delta L, \Delta I$ could not be derived from the other two. In fact, this effect has been reported at least once (by Stevens, 1936). It was the common belief, at that time, that Fechner's conjecture of constant $\Delta L$ was correct. Stevens showed that, when $L$ was obtained from the measurements of B. G. Churcher, and when $\Delta I$ was obtained from the measurements of Riesz, then $\Delta L$ could not be reconciled with Fechner's conjecture. One can show from Stevens's work that for the set of measurements he adopted, $\Delta L \propto$ $L^{0.55}$. So, the result of postulating an inconsistent set led to an inconsistent psychophysical result. Ultimately, a more consistent set was postulated, and psychophysics advanced conceptually.

Our derivation of the equal loudness contours differed from the method of Riesz, whose ratio-of-sums-of-jnds technique was lent support by the work of Lim, Rabinowitz, Braida, and Durlach (1977) and Houtsma, Durlach, and Braida (1980). Ours was a more intuitive technique involving the direct matching of loudness. It did, how- 
ever, require the assumption of an appropriate form for the exponent $n$ as a function of frequency of tone. This method is, therefore, at least in part, empirical, but it does have the advantage of simplicity: It requires only two equations to define the equal loudness contours completely. However, simple does not necessarily mean correct. If further study shows that loudness matching is related to ratios of sums of jnds rather than to sums of jnds, much of the above would have to be modified (see Krueger, 1989 , for expanded discussion).

Returning to Equation 38, the derived contours $\left(I / I_{\text {thresh }}\right)$ are seen to be largely insensitive to the absolute magnitude of $\gamma$ but are sensitive to the ratio of $\gamma$ to $\hat{\gamma}$. For example, if $\gamma$ and $\hat{\gamma}$ were rescaled to be 100 times smaller, the derived contours would remain unchanged. Hence, appealing to Equations 8, 9, and 10, we see that the equal loudness contours are largely insensitive to the form of the individual loudness curves (whether the curve is a power, $\log$, or combination law), since both the power and the log functions emerge from Equation 8 under different values of $\gamma$. It is interesting that the spacing of the contours in equal loudness plots (or the density of contours expressed in contours per unit length along the vertical axis) does depend on the particular form of the loudness function, but the shape of the contours is less dependent.

\section{CONCLUSIONS}

We have tested a more complete form of Fechner's law, which was applied to the sensation of pure tones. It was demonstrated that this form of the law of sensation embraced or contained the usual logarithmic and power functions, but that it offered somewhat more flexibility in the interpretation of psychophysical phenomena. Rather than adhering to a rigid framework of (1) a single, standard law of sensation, (2) a single functional form for the Weber fraction, and (3) a single position on the constancy (or lack thereof) of the subjective jnd, we showed that a unified view of psychophysics would follow from an indefinite number of "triplets" of these three quantities. One must, however, be consistent in the choice of a triplet. The complete form of Fechner's law has a long and variegated history. It may be accepted empirically, or it can be derived from an informational interpretation of the process of perception.

\section{REFERENCES}

BAIRD, J. C., \& Noma, E. (1978). Fundamentals of scaling and psychophysics. New York: Wiley.

BÉKÉsY, G. VoN (1960). Experiments in hearing (E. G. Weaver, Ed. and Trans.). New York: McGraw-Hill.

Delboeuf, J. R. L. (1873). Étude psychophysique: Recherches théoriques et expérimentales sur la mesure des sensations et spécialement des sensations de lumière et de fatigue [Psychophysical study: Theoretical and experimental research on the measurement of sensations and especially of the sensation of light and of fatigue]. In Mémoires couronnés et autres mémoires, publiés parl'Academie Royale des Sciences, des Lettres, et des Beaux-arts de Belgique (Vol. 23, pp. 1-116). Brussels: Hayez.

EkMAN, G. (1959). Weber's law and related functions. Journal of Psychology, 47, 343-352.
Gulick, W. L., Gescheider, G. A., \& Frisina, R. D. (1989). Hearing: Physiological acoustics, neural coding, and psychoacoustics. New York: Oxford University Press.

HARRIS, J. D. (1963). Loudness discrimination. Journal of Speech \& Hearing Disorders: Monograph Supplement (No. 11).

Hellman, R. P., \& ZWISLOCKI, J. (1961). Some factors affecting the estimation of loudness. Journal of the Acoustical Society of America, 33, 687-694.

Hellman, W. S., \& Hellman, R. P. (1990). Intensity discrimination as the driving force for loudness: Application to pure tones in quiet. Journal of the Acoustical Society of America, 87, 1255-1265.

Helm HOLTZ, H. von (1924). Helmholtz's Treatise on physiological optics (Vol. 2; J. P. C. Southall, Ed.). Menasha, WI: Optical Society of America. (Original work published 1856-1866)

Houtsma, A. J. M., Durlach, N. I., \& BRaida, L. D. (1980). Intensity perception XI: Experimental results on the relation of intensity resolution to loudness matching. Journal of the Acoustical Society of America, 68, 807-813.

JesteadT, W., Wier, C. G., \& Green, D. M. (1977). Intensity discrimination as a function of frequency and sensation level. Journal of the Acoustical Society of America, 61, 169-177.

KinG, M. C., \& LockHEAD, G. R. (1981). Response scales and sequential effects in judgment. Perception \& Psychophysics, 30, 599-603.

КоH, K., \& MEYER, D. E. (1991). Function learning: Induction of continuous stimulus-response relations. Journal of Experimental Psychology: Learning, Memory, \& Cognition, 17, 811-836.

KRUEGER, L. E. (1989). Reconciling Fechner and Stevens: Toward a unified psychophysical law. Behavioral \& Brain Sciences, 12, 251 320.

LeHManN, A. (1905). Elemente der Psychodynamik [Elements of psychodynamics]. Leipzig: O. R. Reisland.

Lim, J. S., Rabinowitz, W. M., Braida, L. D., \& Durlach, N. I. (1977). Intensity perception VIII: Loudness comparisons between different types of stimuli. Journal of the Acoustical Society of America, 65, 1256-1267.

MARKs, L. E. (1974a). On scales of sensation: Prolegomena to any future psychophysics that will be able to come forth as science. Perception \& Psychophysics, 16, 358-376.

MARKs, L. E. (1974b). Sensory processes: The new psychophysics. New York: Academic Press.

MARKS, L. E., GAlANTER, E., \& Baird, J. C. (1995). Binaural summation after learning psychophysical functions for loudness. Perception \& Psychophysics, 57, 1209-1216.

MCGiLL, W. J. (1960). The slope of the loudness function: A puzzle. In H. Gulliksen \& S. Messick (Eds.), Psychological scaling: Theory and applications (pp. 67-81). New York: Wiley.

MCGILL, W. J., \& GoldBerG, J. P. (1968). A study of the near-miss involving Weber's law and pure-tone intensity discrimination. Perception \& Psychophysics, 4, 105-109.

MurRaY, D. J. (1993). A perspective for viewing the history of psychophysics. Behavioral \& Brain Sciences, 16, 115-186.

Nicolas, S., Murray, D. J., \& Farahmand, B. (in press). The psychophysics of J. R. L. Delboeuf (1831-1896). Perception.

NORWICH, K. H. (1993). Information, sensation and perception. San Diego: Academic Press.

NoRWICH, K. H., \& WoNG, W. (1995). A universal model of singleunit sensory receptor action. Mathematical Biosciences, 125, 83108.

NutTing, P. G. (1907). The complete form of Fechner's law. Bulletin of the Bureau of Standards, 3, 59-64.

RIESZ, R. R. (1928). Differential intensity sensitivity of the ear for pure tones. Physical Review: Series 2, 31, 867-875.

Robinson, D. W., \& DADSON, R. S. (1956). A re-determination of the equal-loudness relations for pure tones. British Journal of Applied Physics, 7, 166-181.

SCHACKNOW, P., \& RAAB, D. H. (1973). Intensity discrimination of tone bursts and the form of the Weber function. Perception \& Psychophysics, 14, 449-450.

Scharf, B. (1978). Loudness. In E. Carterette \& M. P. Friedman (Eds.), Handbook of perception (Vol. 4, pp. 188-189). New York: Academic Press.

SIEBERT, W. M. (1968). Stimulus transformations in the peripheral au- 
ditory system. In P. A. Kolers \& M. Eden (Eds.), Recognizing patterns (chap. 4, pp. 104-133). Cambridge, MA: M.I.T. Press.

STEvens, S. S. (1936). A scale for the measurement of a psychological magnitude: Loudness. Psychological Review, 43, 405-416.

VIEMEISTER, N. F. (1988). Psychophysical aspects of auditory intensity coding. In G. M. Edelman, W. E. Gall, \& W. M. Cowan (Eds.), Auditory function: Neurobiological bases of hearing (chap. 7, p. 213). New York: Wiley.

WARD, L. M., \& Davidson, K. P. (1993). Where the action is: Weber fractions as a function of sound pressure at low frequencies. Journal of the Acoustical Society of America, 94, 2587-2594.

WEST, R. L. (1996). Constrained scaling: Calibrating individual subjects in magnitude estimation. Published doctoral thesis, University of British Columbia, Vancouver.

WEST, R. L., \& WARD, L. M. (1994). Constrained scaling. In L. M. Ward (Ed.), Proceedings of the Tenth Annual Meeting of the International Society for Psychophysics (p. 225). Vancouver, BC: International Society for Psychophysics.

\section{NOTES}

1. We have shown that $\Delta L$, when computed as the third member of a triplet $(L, \Delta I, \Delta L)$, will often appear as a power function of $L$, by way of mathematical consistency. However, this does not provide an intuitive understanding of the power function relationship. We are aware of only one explanation of this effect that has its basis in neurophysiology. W. S. Hellman and R. P. Hellman (1990) suggested that loudness is a linear function of the mean neural count over a fixed interval of time in a set of auditory nerve fibers. Moreover, they cited experimental evidence to support the thesis that the mean neural count is proportional to the variance of the count in the fibers constituting the set. Using these assumptions and a first-order Taylor series approximation, they were able to derive the equation

$$
\Delta L \propto L^{1 / 2} .
$$

Their calculations were, obviously, approximate, and even a slight change in the mean-variance relation will permit deviations from the exponent of $1 / 2$ to allow for an exponent of $2 / 3$, as derived in Equation 29.
2. It is important to keep in mind the units in which $I$ is measured. If $I$ is measured in units of power [energy/time], $n$ takes on a value of 0.3 at $1000 \mathrm{~Hz}$. However, if $I$ is measured in units of sound pressure [force/ area], then from the relationship $I \propto P^{2}$, we see that $n$ takes on the value 0.6 , or double the previous value. It is easy to confuse the units, as pointed out by Marks (1974a). In this paper, we measure $I$ throughout in units of power.

\section{APPENDIX A}

The Loudness jnd: Constant Versus Nonconstant

The constancy of the subjective jnd (Fechner's famous conjecture) is a necessary condition for expressing the loudness as proportional to the number of jnds. Recall that $\Delta L$ is defined as the change in loudness per $j$ nd. Thus, it may be represented more completely by $\Delta L / \Delta N$, where $\Delta N$ is understood to be 1 jnd. $\Delta L$ now becomes a variable representing the change in loudness required to span $\Delta N=1$ jnd. Thus, the constancy of the jnd may be written

$$
\frac{\Delta L}{\Delta N}=\text { constant }
$$

If we take the liberty of writing $N$, the cumulative number of jnds above threshold, as a continuous variable, then

$$
\frac{d L}{d N}=\text { constant. }
$$

Integrating, we have

$$
L=A N+B,
$$

where $A$ and $B$ are constants. But $L=0$, when $N=0$, which just states that loudness equals zero at threshold. Hence, $B=0$ and

$$
L \propto N .
$$

A

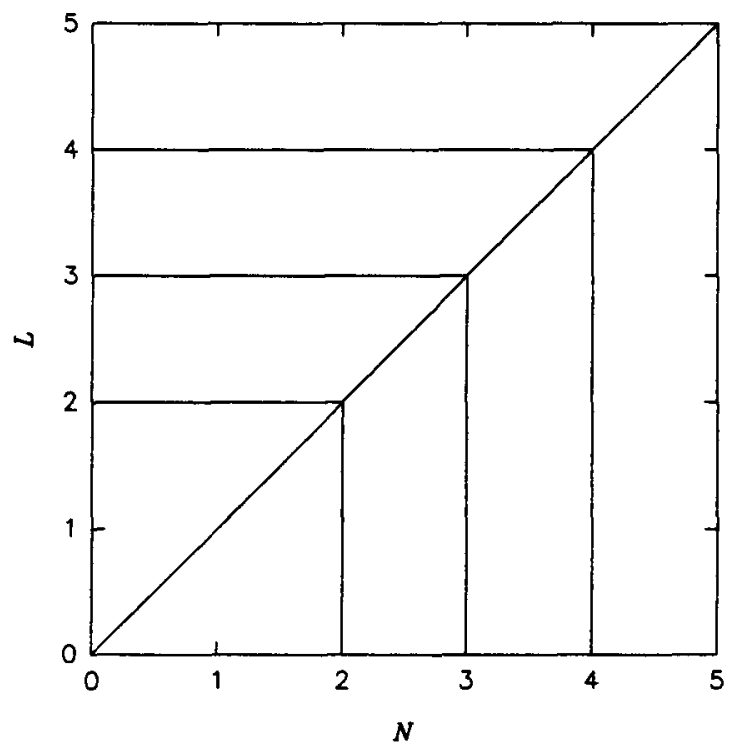

B



Figure A1. (A) Loudness as a sum of jnds. In the case where the subjective jnd is constant, we have loudness proportional to the number of jnds. Equation $A 3$ was plotted with $A=1$ and $B=0$. (B) Loudness as a sum of jnds. This time, the subjective jnd is nonconstant, and the empirical relationship of Stevens (1936), $L=A N^{2.2}$, is plotted with $A=1$. 
Loudness is proportional to number of jnds; loudness is expressible as a sum of loudness jnds.

However, if we replace Equation $\mathrm{Al}$ by the equation

$$
\frac{\Delta L}{\Delta N}=f(N)=\begin{aligned}
& \text { some function of } \\
& \text { cumulative number of jnds, }
\end{aligned}
$$

then, proceeding as before,

$$
L=\int_{0}^{N} f\left(N^{\prime}\right) d N^{\prime} .
$$

$N^{\prime}$ is a dummy variable representing $N$ but used for purposes of integration. For example, we might have $\Delta L / \Delta N=A N^{1.2}$, which upon integration gives $L=A N^{2.2}$, as found by Stevens (1936). $L$ is still equal to the sum of loudness jnds but is not proportional to the number of jnds.

These ideas are represented schematically in Figure A1.

\section{APPENDIX B}

\section{Minimum Audible Field}

To convert between sensation level ( $\mathrm{dB}$ SL) and sound pressure level (dB SPL), we utilize the minimum audible field data
(Gulick, Gescheider, \& Frisina, 1989) of Robinson and Dadson (1956). Their data were fitted to the empirical equation

$$
\begin{aligned}
10 \log _{10}\left(\frac{I_{\text {thresh }}}{I_{o}}\right)= & 3.775\left[\ln \left(\frac{f}{2093}\right)\right]^{2} \\
& +10.53 \exp \left[-\ln ^{2}\left(\frac{f}{1089}\right)\right] \\
& +16.65 \exp \left[-6.343 \ln ^{2}\left(\frac{f}{8295}\right)\right]-8.221
\end{aligned}
$$

The right side of Equation $\mathrm{B} 1$ represents the threshold in units of $\mathrm{dB}$ SPL. To convert between $\mathrm{dB}$ SL and $\mathrm{dB}$ SPL, we use the following equation:

$$
\mathrm{dB} S P L=\mathrm{dB} S P L+\text { threshold. }
$$

(Manuscript received January 16, 1996; revision accepted for publication September 26, 1996.) 